\title{
A Dynamic Model for Ice-Induced Vibration of Structures
}

Department of Engineering Science, Institute of Mechanics,

Chinese Academy of Sciences,

15 Bei Si Huan Xi Road,

Beijing 100080, China

e-mail: ghuang@imech.ac.cn

\section{Pengfei Liu}

Senior Research Officer

Institute for Ocean Technology,

National Research Council Canada,

1 Kerwin Place, P.0. Box 12093,

St. John's, NL, A1B 3T5, Canada

e-mail: pengfei.liu@nrc-cnrc.gc.ca
A dynamic model for the ice-induced vibration (IIV) of structures is developed in the present study. Ice properties have been taken into account, such as the discrete failure, the dependence of the crushing strength on the ice velocity, and the randomness of ice failure. The most important prediction of the model is to capture the resonant frequency lock-in, which is analog to that in the vortex-induced vibration. Based on the model, the mechanism of resonant IIV is discussed. It is found that the dependence of the ice crushing strength on the ice velocity plays an important role in the resonant frequency lock-in of IIV. In addition, an intermittent stochastic resonant vibration is simulated from the model. These predictions are supported by the laboratory and field observations reported. The present model is more productive than the previous models of IIV. [DOI: 10.1115/1.2979795]

Keywords: ice-structure interaction, dynamic modeling, frequency lock-in, resonance, stochastic vibration

\section{Introduction}

Ice-induced vibration (IIV) of structures is an important issue pertaining to the safety of the structures in ice regions, such as offshore drilling platforms, lighthouses, and bridge piers. IIV is harmful to the structural health, which results in the fatigue damage of the structures. More severely, intensive resonant IIV may produce a substantial structural acceleration that exceeds the physical acceptable level for the staff working over them. Therefore, IIV has attracted broad attention from both industrial and academic communities since the 1960s, and much effort has been made to understand the ice-structure interaction process in order to determine the dynamic ice force and structural response. The most important is the mechanism of resonant IIV. Research of IIV is beneficial to the design as well as the operation of the structures.

IIV is a complex dynamic process. The complexity stems from the material and structural failure process of ice, on the one hand, and the nonlinear dynamic coupling of the movements of ice and structures, on the other hand. For a review of current research findings on the ice failure mechanism affecting ice-structure interaction from the sense of ice mechanics, see Dempsey's paper [1] For the case of a vertical slender structure indenting into a moving ice cover, as shown in Fig. 1(a), there have been several analytical IIV models proposed so far. They may be classified into two categories. For the first one, the ice failure is treated as a series of discrete or intermittent events, as proposed by Matlock et al. [2]. This kind of model is applicable to the case of low ice velocity, where the simultaneous ice crushing occurs near the contact area. Matlock's model is simple and rudimentary as it does not take into account some important ice properties. When using Matlock's model, Karr et al. [3] and Trosech et al. [4] found that multiple limiting cycles exist and the structural response is dependent on initial conditions. This indicates that IIV is a complex nonlinear dynamic process. Sodhi [5] extended Matlock's model by introducing an extrusion phase and a possible separation phase, in addition to the loading phase. We call it Matlock-Sodhi model hereafter. The second kind of IIV models treats ice failure as a

Contributed by the Ocean Offshore and Arctic Engineering Division of ASME for publication in the Journal of OfFSHORE MECHANICS AND ARCTIC ENGINEERING. Manuscript received April 22, 2006; final manuscript received June 8, 2008; published December 11, 2008. Assoc. Editor: Walter L. Kuehnlein. Paper presented at the the 25th International Conference on Offshore Mechanics and Arctic Engineering (OMAE2006), Hamburg, Germany, June 4-June 9, 2006. continuous crushing process, such as those proposed by Blenkarn [6] and Määttänen [7]. Based on them, the structure vibrates under self-exciting as a result of dynamic instability due to the negative velocity dependence of the ice crushing strength that appears in the ice velocity region of the ductile-brittle transition. The continuous crushing process may simulate a nonsimultaneous ice crushing at the contact region in the case of high ice velocity, whereas the ductile-brittle transition is produced when the ice velocity is low, at which the simultaneous ice crushing dominates the ice failure process. Indeed, at a high ice velocity, the crushing strength of ice tends to be stable at a relatively low magnitude and the structure vibrates generally in a fashion of high frequency and low amplitude (Peyton [8]). Therefore, research of IIV at low ice velocity is more meaningful, as addressed in this paper.

The aim of the present work is to develop a discrete failure type of dynamic model for IIV by modifying the Matlock-Sodhi model to incorporate more properties of ice crushing, such as the velocity effect and stochastic characteristics of ice crushing. Particular attention is paid to the mechanism of resonant vibration, which threatens the structural safety most remarkably. Our strategy is to consider the main characteristics of ice failure crushing rather than its detailed process. Therefore, the complexity of the model could remain at a treatable level while the salient feature of IIV can be captured. In this paper, we summarize the dynamic property of ice crushing and then describe a new dynamic model. It follows with a presentation and discussion of the deterministic and stochastic structural responses predicted from the present model and some conclusive remarks.

\section{Dynamic Property of Ice Crushing}

It is well known that the ice crushing strength $\sigma_{f}$ (or force $F_{f}$ ) is dependent on the strain or stress rate in the manner as shown schematically in Fig. 2. This experimental finding was obtained from both the laboratory and field tests on either uniaxial compression or indentation samples, referred to Peyton [8], Neill [9], Sodhi $[10,11]$, and Sodhi et al. [12], among others. The dependence curve can be divided into three regions according to ice crushing modes, i.e., ductile, brittle, and middle ductile-brittle transition ones, respectively. In the ductile mode, ice crushes by microcracking and macrosplitting through the propagation of cleavage cracks in a fashion of simultaneous failure. While in the brittle mode, nonsimultaneous crushing occurs through microcracking, shearing, extrusion, and flaking, with high-pressure zones formed on the contact surface. 

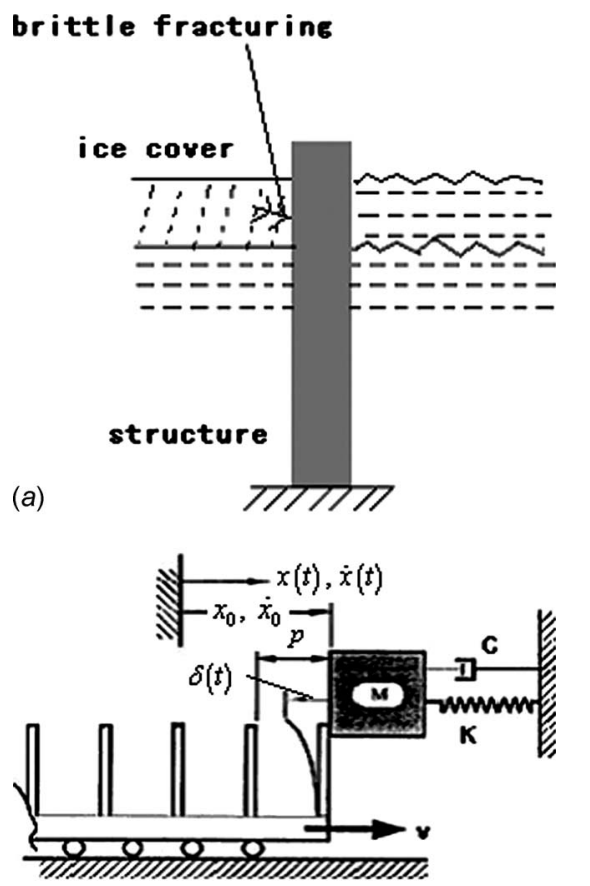

(b)

Fig. 1 Dynamic model for ice-structure interaction

The strain rate is often defined by $v_{r} / h$ or $v_{r} / D$, where $v_{r}$ is the ice velocity relative to the structure, $h$ is the thickness of the ice cover, and $D$ is the diameter of the structure. Thus, for the prescribed ice cover and structure, the dependence of the ice crushing strength on the strain rate is equivalent to that on the relative velocity. Following Iliescu and Schulson [13], the relationship of ice crushing strength versus strain rate may be expressed by two separate power laws according to when the strain rate $\dot{\varepsilon}$ is below or above the transitional strain rate $\dot{\varepsilon}_{t}=v_{t} / h$ corresponding to the maximum strength $\sigma_{f \max }$, as indicated in Fig. 2, where $v_{t}$ is the transitional relative ice velocity. Therefore, the ice crushing strength curve may be expressed, in a dimensionless form, as

$$
\bar{\sigma}_{f}= \begin{cases}\left(1-\bar{\sigma}_{f d}\right)\left(v_{r} / v_{t}\right)^{\alpha}+\bar{\sigma}_{f d}, & v_{r} / v_{t} \leq 1 \\ \left(1-\bar{\sigma}_{f b}\right)\left(v_{r} / v_{t}\right)^{\beta}+\bar{\sigma}_{f b}, & v_{r} / v_{t}>1\end{cases}
$$

where $\bar{\sigma}_{f}=\sigma_{f} / \sigma_{f \max }, \bar{\sigma}_{f d}=\sigma_{f d} / \sigma_{f \max }$, and $\bar{\sigma}_{f b}=\sigma_{f b} / \sigma_{f \max }$, with $\alpha>0$ and $\beta<0$ being indices and $\sigma_{f d}$ and $\sigma_{f b}$ are the minimum ice crushing strengths in the ductile and brittle regions, respectively, as defined in Fig. 2.

\section{Dynamic Model of Ice-Structure Interaction}

Like Matlock's model, only the fundamental modal response of the structure is considered in the present model, as the first order approximation. Then the structure can be simulated as an oscilla-

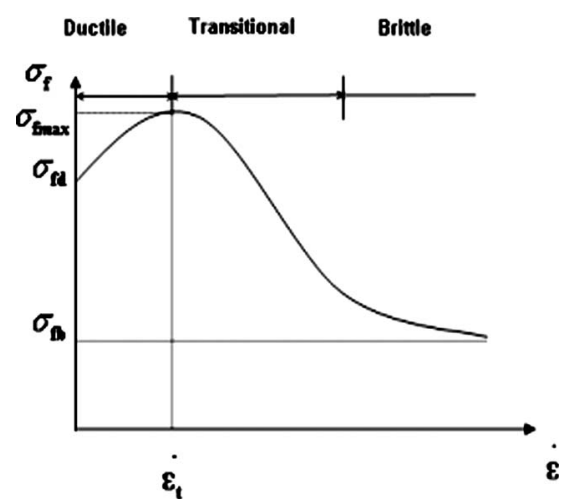

Fig. 2 Characteristic plot of ice crushing strength versus strain rate

tor consisting of a mass $M$, a viscous dashpot with damping coefficient $C$, and a linearly elastic spring of stiffness $K$, as shown in Fig. 1(b). Included also is the ice cover model consisting of a series of ice teeth to simulate the discrete failure mode in the ductile and transitional regions. In Fig. 1(b), the position and the velocity of the mass are denoted by $x$ and $\dot{x}$, respectively, with the corresponding initial values being $x_{0}$ and $\dot{x}_{0} ; v$ is the velocity of the ice cover and the distance between adjacent teeth, $p$ represents the length of the failure zone associated with each failure event and is assumed to be constant, independent of the ice velocity.

The deflection of the tooth $\delta(t)$ is

$$
\delta(t)=x_{0}+v t-x(t)-p(n-1)
$$

where $t$ is the time and $n$ is the number of the tooth in contact. After the ice cover and structure come into contact, ice force $F$ is built up until it reaches the failure force $F_{f}$. This process is called loading phase during which the contact between the mass and ice cover is assumed to be linearly elastic with an effective contact stiffness $k$. The ice force $F$ is then related to $\delta$ through $F=k \delta$ $+F_{e}$ in this loading phase, where $F_{e}$ is the residual force remained during the previous crushing event. Although it may be more realistic to take a nonlinear relationship between $F$ and $\delta$, considering the microdamaging during the loading phase, the above linearization through introducing the effective contact stiffness keeps the qualitative characteristic of the structural response unchanged since the nonlinearity results mainly from the intermittent ice failure events. After the ice failure occurs, the mass penetrates through the crushed ice and extrudes it until the next tooth comes into contact. This is an extrusion phase during which the ice force is assumed to be constant and identical to $F_{e}$. At this phase, $\delta$ represents only the relative displacement between the ice and structure. During both the loading and extrusion phases, the separation of the mass and the ice cover may occur if the velocity of the mass is greater than the ice velocity. Therefore, $F$ vanishes in this separation phase.

Based on the above analysis, the governing equation of motion for the mass is given as

$$
M \ddot{x}+C \dot{x}+K x= \begin{cases}k\left[x_{0}+v t-x-p(n-1)\right]+F_{e}, & 0 \leq \delta<\delta_{f} \text { and } \dot{x} \leq v \\ F_{e}, & \delta_{f} \leq \delta<p \text { and } \dot{x} \leq v \\ 0, & \dot{x}>v\end{cases}
$$

where $\ddot{x}=d^{2} x / d t^{2}$ is the acceleration of the mass and $\delta_{f}=\left(F_{f}-F_{e}\right) / k$ is the failure deflection. Let us introduce the following normalized quantities:

$$
\begin{gathered}
\bar{x}=x / \Delta, \quad \bar{x}_{0}=x_{0} / \Delta, \quad \bar{p}=p / \Delta, \quad \bar{\delta}=\delta / \Delta, \quad \bar{\delta}_{f}=\delta_{f} / \Delta \\
\tau=\omega_{n} t, \quad \bar{k}=k / K, \quad \bar{F}_{e}=F_{e} / F_{f \max }, \quad \bar{v}=v /\left(\omega_{n} \Delta\right)
\end{gathered}
$$




$$
\bar{v}_{t}=v_{t} /\left(\omega_{n} \Delta\right)
$$

in which

$$
\Delta=\frac{F_{f \max }}{K} \quad \text { and } \quad \omega_{n}=\sqrt{\frac{K}{M}}
$$

are the maximum displacement of the mass corresponding to the static application of the maximum failure ice force $F_{f}$ max and the natural angular frequency of the structure, respectively. Then Eq. (3) can be nondimensionalized as

$$
\ddot{\bar{x}}+2 \xi \dot{\bar{x}}+\bar{x}=\left\{\begin{array}{lll}
\bar{k}\left[\bar{x}_{0}+\bar{v} \tau-\bar{x}-\bar{p}(n-1)\right]+\bar{F}_{e}, & 0 \leq \bar{\delta}<\bar{\delta}_{f} \text { and } \quad \dot{\bar{x}} \leq \bar{v} \\
\bar{F}_{e}, & \bar{\delta}_{f} \leq \bar{\delta}<\bar{p} \text { and } \dot{\bar{x}} \leq \bar{v} \\
0, & \dot{\bar{x}}>\bar{v} &
\end{array}\right.
$$

where $\xi=C / 2 M \omega_{n}$ is the damping ratio, $\ddot{\bar{x}}=d^{2} \bar{x} / d \tau^{2}$, and $\dot{\bar{x}}$ $=d \bar{x} / d \tau$.

From the definition of $\delta_{f}$ and Eqs. (4) and (5), we get

$$
\bar{\delta}_{f}=\frac{\left(\bar{F}_{f}-\bar{F}_{e}\right)}{\bar{k}}
$$

with $\bar{F}_{f}=F_{f} / F_{f \text { max }}$. Following Korzhavin's formula for ice force [14], the ice failure force is related to the ice failure strength by

$$
F_{f}=\operatorname{Im} \kappa h D \sigma_{f}
$$

where $I, m$, and $\kappa$ are the indentation, geometry, and contact coefficients, respectively. Hence, it is obtained readily that $\bar{F}_{f}=\bar{\sigma}_{f}$ and then

$$
\bar{\delta}_{f}\left(v_{r} / v_{t}\right)=\frac{\left[\bar{\sigma}_{f}\left(v_{r} / v_{t}\right)-\bar{F}_{e}\right]}{\bar{k}}
$$

from Eqs. (7) and (1), in which $v_{r}=v-\dot{x}$. Recently, Tong et al. [15] observed, from the laboratory test on the indentation of a rigid structure into an ice cover, that the ice failure frequency increased linearly with the ice velocity until $v / h=5.4 \times 10^{-1} / \mathrm{s}$. Since it covers the typical ductile and transitional regions and the ice failure frequency is given by $f_{\text {ice }}=v / p$ for rigid structures, it means that $p$ can be considered to be independent of the ice velocity in the low-velocity region, which is concerned with in the present model.

Equation (6) is a highly nonlinear dynamic equation, owing to the discrete failure events and the dependence of the ice failure force on the relative ice velocity. However, it is piecewise linear and, hence, a closed form solution can be derived for the individual phase. They are the following.

(a) In the loading phase,

$$
\bar{x}=e^{-\xi \tau_{l}}\left\{\begin{array}{l}
{\left[\bar{x}_{0 l}\left(1-k_{r}\right)-\left(\bar{F}_{e}-2 \xi k_{r} \bar{v}\right) / k_{q}^{2}\right] \cos \omega_{l} \tau_{l}+} \\
\frac{1}{\omega_{l}}\left[\dot{\bar{x}}_{0 l}+\xi \bar{x}_{0 l}\left(1-k_{r}\right)-\left\langle\xi \bar{F}_{e} / k_{q}^{2}+k_{r} \bar{v}\left(1-2 \xi^{2} / k_{q}^{2}\right)\right\rangle\right] \sin \omega_{l} \tau_{l}
\end{array}\right\}+\bar{F}_{e} / k_{q}^{2}+k_{r}\left(\bar{x}_{0 l}+\bar{v} \tau_{l}-2 \xi \bar{v} / k_{q}^{2}\right)
$$

where $\tau_{l}=\tau-\tau_{0 l}, \omega_{l}^{2}=k_{q}^{2}-\xi^{2}, k_{q}^{2}=1+\bar{k}$, and $k_{r}=\bar{k} / 1+\bar{k} ; \tau_{0 l}, \bar{x}_{0 l}$, and $\dot{\bar{x}}_{0 l}$ are the initial values of the time, position, and velocity, respectively, at the phase.

(b) In the extrusion phase,

$$
\begin{aligned}
\bar{x}= & e^{-\xi \tau_{e}}\left\{\left(\bar{x}_{0 e}-\bar{F}_{e}\right) \cos \omega_{e} \tau_{e}+\frac{1}{\omega_{e}}\left[\dot{\bar{x}}_{0 e}+\xi\left(\bar{x}_{0 e}\right.\right.\right. \\
& \left.\left.\left.-\bar{F}_{e}\right) \sin \omega_{e} \tau_{e}\right]\right\}+\bar{F}_{e}
\end{aligned}
$$

where $\omega_{e}^{2}=1-\xi^{2}$ and $\tau_{e}=\tau-\tau_{0 e} ; \tau_{0 e}, \bar{x}_{0 e}$, and $\dot{\bar{x}}_{0 e}$ are the initial values of the time, position, and velocity, respectively, at the phase.

(c) In the separation phase,

$$
\bar{x}=e^{-\xi \tau_{s}}\left[\bar{x}_{0 s} \cos \omega_{e} \tau_{s}+\frac{1}{\omega_{e}}\left(\dot{\bar{x}}_{0 s}+\xi \bar{x}_{0 s}\right) \sin \omega_{e} \tau_{s}\right]
$$

where $\tau_{s}=\tau-\tau_{0 s} ; \tau_{0 s}, \bar{x}_{0 s}$, and $\dot{\bar{x}}_{0 s}$ are the initial values of the time, position, and velocity, respectively, at the phase.
The complete solution to Eq. (6) is an alternative combination of Eqs. (10)-(12), with the initial time, position, and velocity of each phase determined by the contact, ice failure, and separation conditions, respectively, as indicated in Eq. (6). Since they depend on the instantaneous relative motion between the structure and ice, as prescribed by Eqs. (9) and (1), the numerical computation is needed to obtain the dynamic structural response and the associated ice force time histories.

\section{Steady Response and Ice Force}

For the limitation of the paper length, we highlight the influence of the ice velocity on the structural vibration characteristics. The structure and ice properties used in the computation are

$$
\bar{k}=0.1, \quad \xi=0.04, \quad \bar{\sigma}_{f d}=0.7, \quad \bar{\sigma}_{f b}=0.5
$$




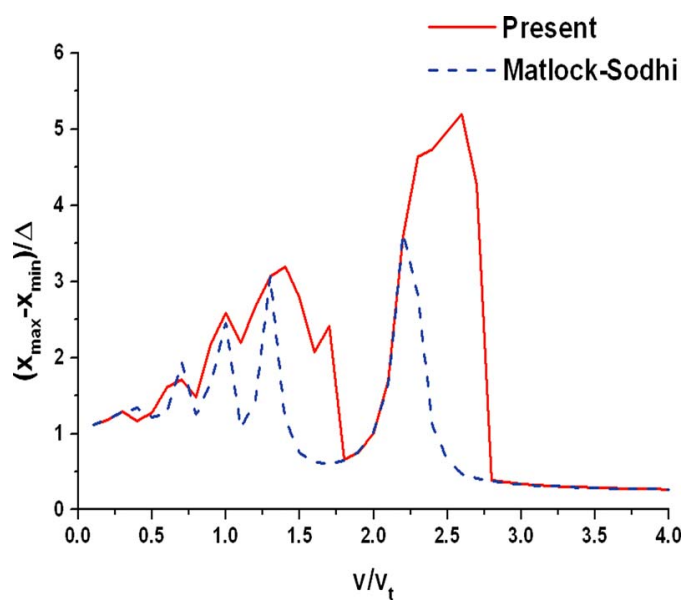

Fig. 3 Dependence of the amplitude of the structural vibration on the ice velocity

$$
\alpha=0.5, \quad \beta=-2, \quad \bar{p}=10, \quad \bar{F}_{e}=0.2, \quad \bar{v}_{t}=1
$$

which lead to $p=\delta_{f \max }$ since $p=\bar{p} \bar{k} \delta_{f \text { max }}$ from Eqs. (4) and (5).

Figure 3 shows the dimensionless amplitude of the steady structural vibration $\bar{x}_{\max }-\bar{x}_{\min }$ versus the dimensionless ice velocity $v / v_{t}$ predicted from the present model and the Matlock-Sodhi model, respectively. The former reduces to the latter when $\bar{F}_{f}$ $=\bar{\sigma}_{f}$ is set constant. In the calculations, the constant $\bar{F}_{f}$ for the Matlock-Sodhi model is given by Eq. (1) by setting $v_{r}$ equal to $v$ as an average. It is noted that the velocity effect is so appreciable that the two models predict either nearly the same or much different amplitude of the structural vibration, depending on the range of the ice velocity. If we confine ourselves to the maximum peak in the curve corresponding to the Matlock-Sodhi model, as plotted by the dashed line, it can be seen that at an ice velocity of $v / v_{t}$ falling into the ascending portion of the peak or into the portion above about 2.8 , the two models are almost indistinguishable. The difference of the predictions is remarkable otherwise, for $v / v_{t}$ being over 2.2-2.75, which is just the descending portion of the peak. The maximum difference achieves as high as one order of magnitude over that velocity range.

To further clarify the underlying mechanism leading to the above difference, comparison is made of their profiles of the steady structural response and ice force time histories, as indicated in Fig. 4 and 5 at $v / v_{t}=2.2$ and 2.6, respectively. They are the limiting cycles, as the attractors in the phase plot. At $v / v_{t}=2.2$, the velocity corresponding to the top of the peak, the limiting cycles for the two predictions are the same both in amplitude and in phase, i.e., there exist one deflection cycle and one ice failure event per period, as shown in Fig. 4. However, at $v / v_{t}=2.6$, which corresponds to the maximum difference between the two predictions, there are nine deflection cycles and ten ice failure events per period in the present prediction, instead of one deflection cycle and one ice failure event per period in the prediction based on the Matlock-Sodhi model, as shown in Figs. 5(a) and 5(b). Figure $5(a)$ shows that the peak vibration amplitude occurs when the ice force and the structural response are in phase. As the amplitude of vibration increases gradually, ice failure events occur when the structure and ice move in the same direction with the structure gaining momentum from the ice. In contrast, as the amplitude of vibration decreases gradually, ice failure events occur when the structure and ice move in an opposing direction with the ice gaining momentum from the structure. This is the typical feature of the resonant vibration, as observed by Engelbrektson [16] in the Gulf of Bothnia. Further computation has shown that this feature is dominant when $v / v_{t}$ is between over 2.2 and 2.75 .

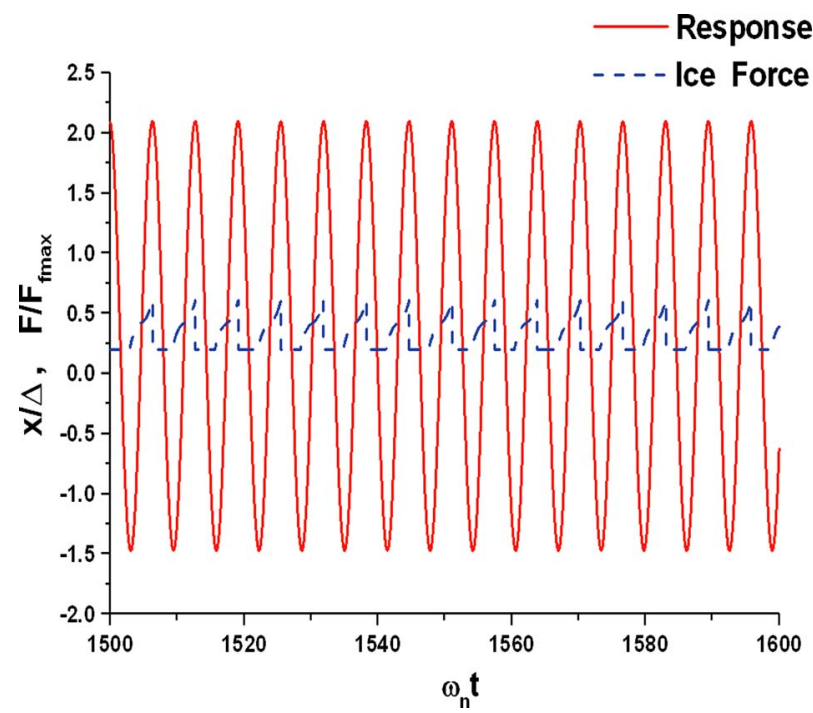

Fig. 4 Response and ice force time history plots predicted from the present or Matlock-Sodhi model. The two models give the same prediction: $v / v_{t}=2.2, \quad \bar{x}_{0}=0$ and $\dot{\bar{x}}_{0}=0$.

\section{Resonant Frequency Lock-In}

The resonance feature may be further demonstrated by the spectrum analysis. Figure 6 shows the predominant frequency of the ice force in the amplitude spectrum as a function of the ice velocity based on Fourier analysis. The corresponding predominant frequency of the response is nearly the same. It turns out that in the concerned region of $v / v_{t}$, over 2.2-2.75, the frequency of the ice force is locked on the natural frequency of the structure $f_{n}=\omega_{n} / 2 \pi$, as the solid line shows, leading to an intensive vibration. Included also in Fig. 6 is the related result based on the Matlock-Sodhi model, as the dashed lines show. Observing that for $v / v_{t}$ over 2.0-2.2 or above 2.75, the predominant frequencies predicted from the two models are almost the same (the solid and dashed lines almost coincide), in consistence with the corresponding responses shown in Fig. 3 and 4. However, at $v / v_{t}=2.2$, a new attractor of resonance begins to appear due to the bifurcation of nonlinear dynamics and the original attractor becomes unstable until $v / v_{t}=2.6$, as indicated by the dashed lines. Therefore, there exists a transition region between $v / v_{t}=2.6$ and 2.75 in which there are two attractors and the structural response of the structure is attracted by either of them, depending on the initial conditions $\bar{x}_{0}$ and $\dot{\bar{x}}$. It is found that at $v / v_{t}=2.6$, for instance, when $\bar{x}_{0}=0$ and $\dot{\bar{x}}=0$ are set, the corresponding attractor is just the same as Fig. $5(b)$, which is called the Matlock-Sodhi type of attractor, while when $\bar{x}_{0}=0.5$ and $\dot{\bar{x}}=0$ are set, the corresponding attractor is just the same as Fig. 5(a), featuring resonance. After $v / v_{t}=2.75$ is reached, the attractor of resonance becomes unstable and only the Matlock-Sodhi type of attractor remains. Määttänen [17] observed the resonant frequency lock-in at a wide velocity range. It is interesting to note that the resonant frequency lock-in in IIV is quite similar to that in vortex-induced vibrations (VIVs), referred to the experimental result of VIV (Feng [18]). This means that there is a dynamic analogy between IIV and VIV, although their natures are completely different. This is possibly because both the ice failure and the vortex-shedding are intermittent processes.

We may now give an explanation on the mechanism of resonant IIV, based on the above dynamic analysis. The ice-structure interaction plays dual roles in IIV, promoting and restraining the vibration of structures, as illustrated above. However, the relative domination of the two roles is uneven for compliant structures, even if the velocity effect on the ice failure force is not involved. Due to the movement of the structure, the relative ice velocity is 

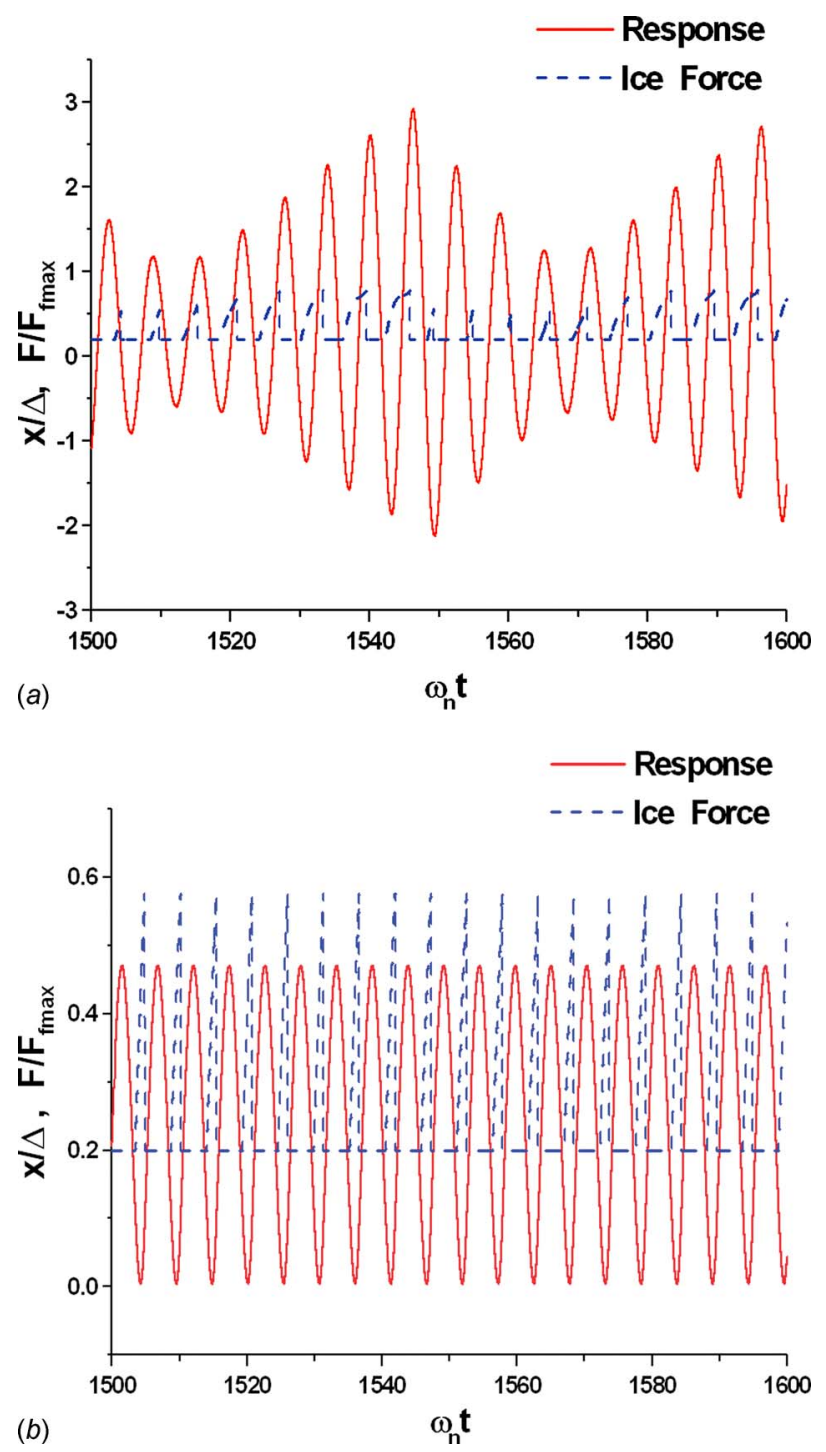

Fig. 5 Response and ice force time history plots predicted from the present model (a) and Matlock-Sodhi model (b). $v / v_{t}$ $=2.6, \bar{x}_{0}=-0.5$, and $\dot{\bar{x}}_{0}=0$.

smaller when the structure and ice move in the same direction than in the opposing direction. Thus, the buffering action takes effect in the former case to lengthen the duration of ice-structure interaction before ice failure occurs and so the transmission of momentum or energy from ice to the structure is more than the inverse transmission in the latter case. This unevenness causes a net gain of energy for the structure, which is balanced by the damping dissipation and the potential and dynamic energies of the vibration in the steady vibration state. The velocity effect on the ice failure force further signifies the unevenness since not only the loading phase lengthens but also the ice force during the interaction increases when the ice and structure moves in the same direction, as illustrated by the ice force time history plot in Fig. $5(a)$. Consequently, the rate of the net energy gain increases so that the system stays in the dynamic equilibrium with higher amplitude of response. The resonant frequency lock-in maximized the net gain rate of energy. In addition, it is noted that a higher rate of net energy gain is produced when the ice velocity falls into such a range that the relative ice velocity remains in the descending portion of the ductile-brittle transition region, irrespective of the movement direction of the structure. This is because the alternation of the ice failure force is most remarkable in this range.

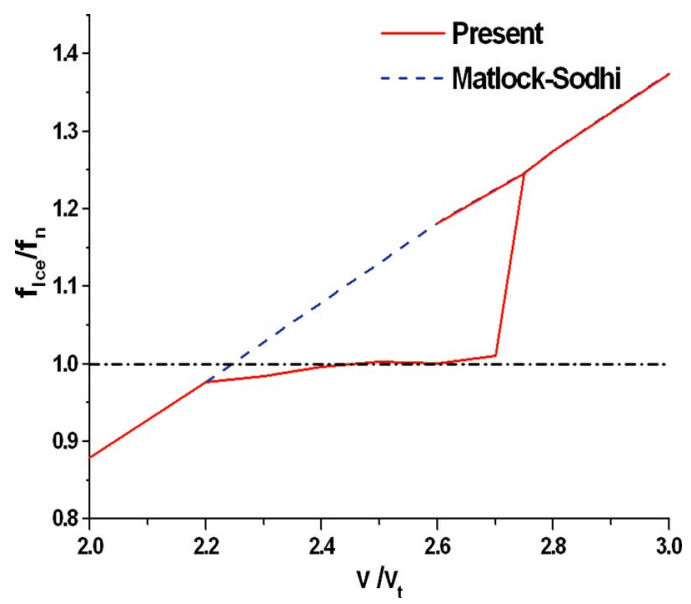

Fig. 6 Lock-in of the ice force frequency to the natural frequency of the structure in the ice velocity region of resonant vibration

Therefore, it explains why the ice velocity range of the frequency lock-in in Fig. 6 shifts to the right in a distance from the ductilebrittle transition region.

\section{Stochastic Effect of Ice Failure on IIV}

Randomness is the intrinsic characteristic of the fracture and failure of brittle materials, particularly in dynamic cases. Therefore, we need to further incorporate the randomness of ice failure properties into the present model. In the following numerical computation, $10 \%$ of the randomness with uniform probabilistic distribution imposed on $\bar{F}_{f}$ and $\bar{p}$ is considered.

Figure 7 shows the stochastic response of the structure at ice velocities of $v / v_{t}=2.5$. The detailed response and ice force time history plots for two typical phases in Fig. 7 are shown in Figs. $8(a)$ and $8(b)$. Comparing them with Fig. 5, it is noted that a stochastic resonance appears in an intermittent way that the system migrates between the resonant attractor and the MatlockSodhi type of attractor and oscillates stochastically near them. Meanwhile, our computation shows that at $v / v_{t}=2.4$, only the stochastic resonant vibration appears. The intermittent resonant vibration predicted here is quite alike to the field observation by Engelbrektson [16]. It was reported that during most of the day, the acceleration was below $0.07 \mathrm{~g}$ (gravity acceleration), while it was as high as $0.7 \mathrm{~g}$ when the resonant vibration occurred.

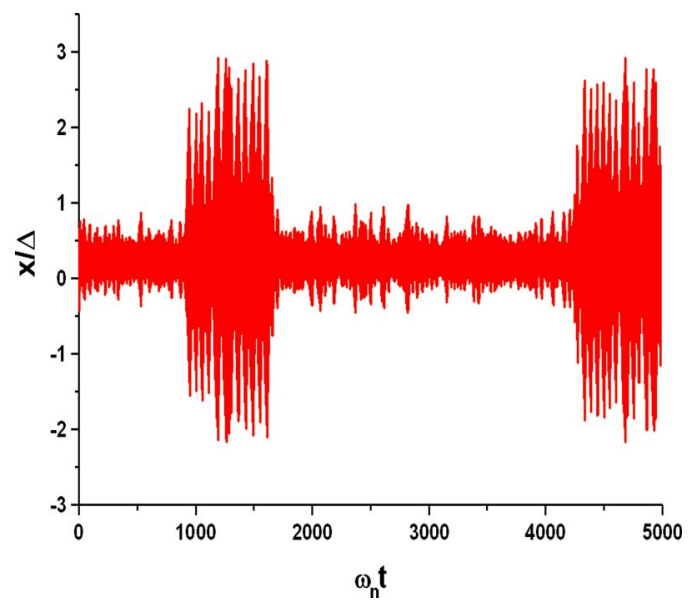

Fig. 7 Stochastic structural responses: $v / v_{t}=2.5$ 

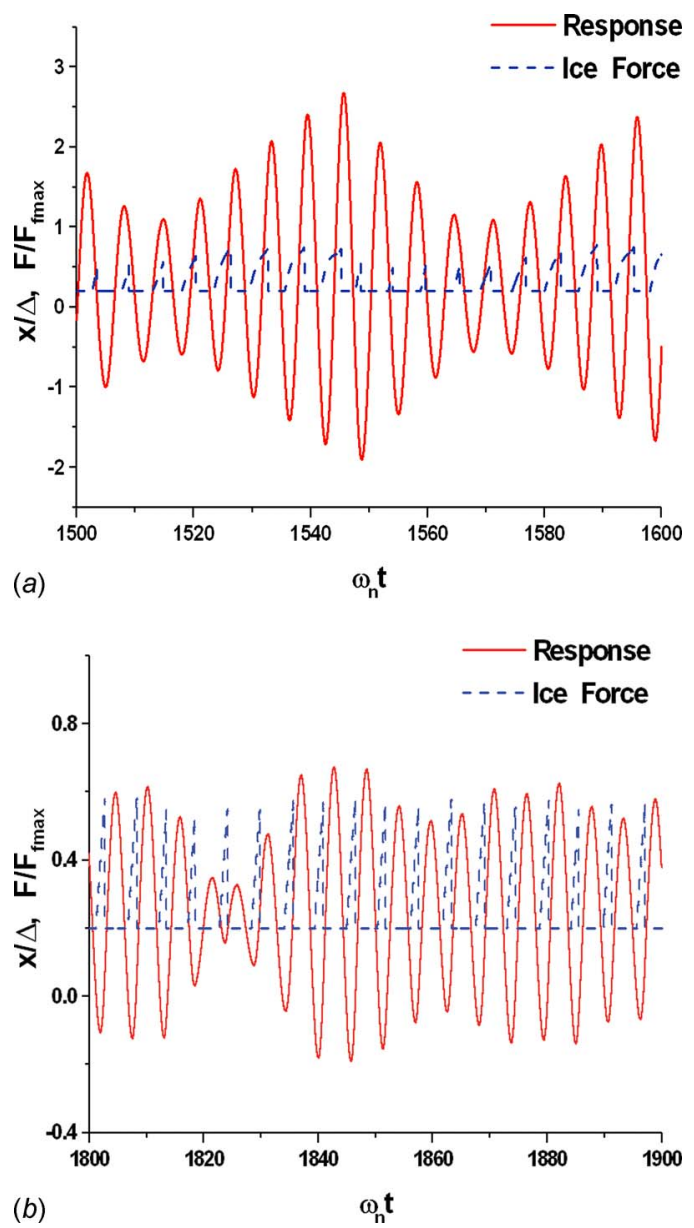

Fig. 8 Detailed ice force and response time history plots corresponding to two typical phases in Fig. 7

\section{Conclusive Remarks}

In the present study, a dynamic model for IIV has been developed. Some ice properties have been taken into account, such as the discrete failure, the dependence of the crushing strength on the ice velocity, and the randomness of the ice failure. The model captures resonant frequency lock-in, which is the most important feature of IIV. The predicted results reasonably explain the mechanism of resonance. In addition, the present model predicts the intermittent resonant vibration. These theoretical results are supported by the laboratory and field observations reported.

\section{Acknowledgment}

The authors thank for the financial supports from the Chinese Academy of Sciences in terms of Key Project of Innovation Program and from the National Natural Science Foundation of China (Grant Nos. 10772184 and 10532070). The authors also thank the National Research Council Canada for its support.

\section{References}

[1] Dempsey, J. P., 2000, "Research Trends in Ice Mechanics," Int. J. Solids Struct., 37, pp. 131-153.

[2] Matlock, G., Dawkins, W., and Panak, J., 1971, "Analytical Model for IceStructure Interaction," J. Engrg. Mech. Div., EM4, pp. 1083-1092.

[3] Karr, D. G., Troesch, A. W., and Wingate, W. C., 1992, "Nonlinear Dynamics Response of a Simple Ice-Structure Interaction Model," Proceedings of the 11th International OMAE Conference, June 7-11, 1992, Vol. IV, pp. 231-237.

[4] Troesch, A. W., Karr, D. G., and Beier, K. P., 1992, "Global Contact Dynamics of an Ice-Structure Interaction Model," Int. J. Bifurcation Chaos Appl. Sci. Eng., 2, pp. 607-620.

[5] Sodhi, D. S., 1994 "A Theoretical Model for Ice-Structure Interaction," Proceedings of the OMAE-94 Conference, February 27-March 3, 1994, ASME, New York, Vol. IV, pp. 29-34

[6] Blenkarn, K. A., 1970, "Measurement and Analysis of Ice Force on Cook Inle Structures," Proceedings of the Second Offshore Technology Conference (OTC) No. 1261, Dallas, TX, April 22-24, 1970, Vol. II, pp. 365-378.

[7] Määttänen, M., 1978, "On Conditions for the Rise of Self-Excited Ice Induced Autonomous Oscillations in Slender Marine Pile Structures [R]," FinnishSwedish Winter Navigation Research Board, Research Report No. 25.

[8] Peyton, H. R., 1968, "Ice and Marine Structure, Part II: Sea Ice Properties," Ocean Indus., 3, pp. 59-65.

[9] Neill, C., 1976, "Dynamic Ice Forces on Piers and Piles: An Assessment of Design Guidelines in the Light of Recent Research," Can. J. Civ. Eng., 3, pp. 305-341.

[10] Sodhi, D. S., 1998, "Nonsimultaneous Crushing During Edge Indentation of Freshwater Ice Sheets," Cold Regions Sci. Technol., 27, pp. 179-195.

[11] Sodhi, D. S., 2001, "Crushing Failure During Ice-Structure Interaction,” Eng. Fract. Mech., 68, pp. 1889-1921.

[12] Sodhi, D. S., Takeuchi, T., Nakazawa, N., Akagawa, S., and Saeki, H., 1998, "Medium-Scale Indentation Tests on Sea Ice at Various Speeds," Cold Regions Sci. Technol., 28, pp. 161-182.

[13] Iliescu, D., and Schulson, E. M., 1999, "Brittle Compressive Failure of Ice: Monotonic Versus Step-Loading," Proceedings of the 18th International OMAE Conference CD-ROM: (OMAE99-1170), July 11-16, 1999, p. 14

[14] Korzhavin, K. N., 1962, "Action of Ice on Engineering Structures," U.S Army Cold Regions Research and Engineering Laboratory, CRREL Draft Translation No. 260.

[15] Tong, J. F., Song, A., and Shi, Q. Z., 2001, "An Experimental Study of IceInduced Vibration and Dynamic Characteristics of Ice Loads," Ocean Eng., 19, pp. 34-39.

[16] Engelbrektson, A., 1983, "Observations of a Resonance Vibrating Lighthouse Structure in Moving Ice," Proceedings of the Seventh POAC Conference, Helsinki, Finland, April 5-9, 1983, Vol. II, pp. 855-864.

[17] Määttänen, M., 1983, "Dynamic Ice Structure Interaction During Continuous Crushing," U.S Army Cold Regions Research and Engineering Laboratory, CRREL Report No. 83-5.

[18] Feng, C. C., 1968, "The measurement of Vortex-Induced Effects in Flow Past Stationary and Oscillating Circulator and D-Section Cylinder," M.S. thesis, University of British Columbia, Vancouver. 